\title{
Large-eddy simulation of flow and combustion dynamics in a lean partially premixed swirling combustor
}

\author{
Shaoshuai Li ${ }^{\mathrm{a}}$, Yunzhe Zheng ${ }^{\mathrm{a}}$, Min Zhu ${ }^{\mathrm{a}}$, Daniel Mira Martinez ${ }^{\mathrm{b}}$, Xi Jiang ${ }^{\mathrm{b}}$ \\ ${ }^{a}$ Key Laboratory for Thermal science and Power Engineering of Ministry of Education, \\ Department of Thermal Engineering, Tsinghua University, Beijing, 100084, China \\ ${ }^{b}$ Department of Engineering, Lancaster University, Lancaster, LA1 4YR, UK
}

\begin{abstract}
A lean partially premixed swirling combustor was studied by resolving the complete flow path from the swirl vanes to the chamber outlet with large-eddy simulation (LES). The flow and combustion dynamics for non-reacting and reacting situations was analysed, where the intrinsic effects of swirl vanes and counter flow on the vortex formation, vorticity distribution for non-reacting cases were examined. A modified flame index was introduced to identify the flame regime during the partially premixed combustion. The combustion instability phenomenon was examined by applying Fourier spectra analysis. Several scalar variables were monitored to investigate the combustion dynamics at different operating conditions. The effects of swirl number, Reynolds number, equivalence ratio and nitrogen dilution on combustion dynamics and NOx emissions were found to be significant.
\end{abstract}

Keywords: LES, lean partially premixed, swirling flows, combustion dynamics, $\mathrm{NO}_{\mathrm{X}}$

\section{Introduction}

Lean premixed (LP) combustion is a promising technology used in gas turbine engines for reducing both $\mathrm{NO}_{\mathrm{X}}$ emissions as well as reaction zone size. To obtain optimal fuel/air mixing at the combustor inlet and avoid combustion instability, such as flame quenching or flashback during LP combustor operation, swirling injectors are typically used in gas turbine systems

\footnotetext{
*Corresponding author.Tel:+86 13810086132

Email address: zhumin@mail.tsinghua.edu.cn (Min Zhu)
} 
to provide the desired fuel/air distribution and produce central toroidal recirculation zones (CTRZs), which serve as the dominant flame stabilization mechanism $^{1}$. However, the streamwise and spanwise vortices breakdown due to swirl flows give rise to high shear layer instability and turbulence intensity, which may cause flow motion instability and couple with acoustic waves to generate combustion oscillation ${ }^{2}$.

Many works have been conducted to study the influence of intrinsic swirling flow on combustion instability. Huang ${ }^{3}$ analysed the effect of swirl number on flow development and combustion dynamics in a lean premixed combustor. Results indicated that excessive swirl caused the recirculation zones to move upstream and may result in flame flashback. Fernandes ${ }^{4}$ conducted experiments to investigate the swirl effect on flow instability characteristics and found that the instability frequency was determined by the axial translation of spiral vortex while the angular transportation of the vortex core was the dominant factor when the swirl number exceeded 0.88 ; the instability frequency varied parabolically with the swirl number. Sommerer et al. ${ }^{5}$ studied the transition of different combustion regimes using both experimental and the LES methods in a partially premixed swirling burner and discovered the critical role of swirl and recirculation zones during flame flashback combustion regime. Weigand et al. ${ }^{6}$ conducted a detailed experiment that measured three types of swirl methane flames with different combustion instability characteristics. Flow field, vortex structure and reaction species were carefully analysed. Meier ${ }^{7}$ analysed the effects of turbulence flow on the thermal chemistry state and the structures of $\mathrm{CH}$ layers in a partially premixed swirling combustor by applying planar laser-induced fluorescence (PLIF), laser Doppler velocimetry (LDV) and Raman scattering. Results indicated that swirl flames behaved more like a diffusion flame than a uniformly premixed flame because of the existence of a spatial gradient in the mixture fraction and the larger widths of the $\mathrm{CH}$ layers compared to premixed flames.

Several other works have focused on the effects of different operating conditions on flame dynamics. Huang and Yang ${ }^{8,9}$ investigated the effects of inlet flow conditions on unsteady flame dynamics. Several influencing factors were identified and analysed. Prakash et al. ${ }^{10}$ studied flame dynamics at different equivalence ratios and developed an efficient method for preventing lean blow out. Menon et al. ${ }^{11}$ simulated a typical GE turbine engine, model LM6000, with the LES method and investigated the influence of swirl number and a subgrid model on the mixing and reaction processes. Schluter ${ }^{12}$ focused on developing a combustion oscillation control method and investi- 

5.

gated the effects of large-scale vortices on combustion dynamics in a swirl coaxial combustor. Grinstein ${ }^{13}$ examined the effects of combustor confinement geometry on flame and flow characteristics in a swirl model combustor. Duwig $^{14}$ detected the flame stabilization factor in a swirl-stabilized combustor.

Although valuable information has been obtained by these studies, the flame dynamics and instability mechanism are very complex at lean conditions considering the existence of swirling flow. While many works have been conducted to study such mechanisms under lean premixed operating conditions, LES investigation for lean partially premixed operating conditions is relatively rare. There are still many unresolved issues regarding flame dynamics, $\mathrm{NO}_{\mathrm{x}}$ emissions and the mechanisms of flame/vortex interactions in swirl lean partially premixed combustors, which require further investigation. In the present work, a swirl-stabilized lean partially-premixed combustor in our experiment is simulated using a parallel LES method to examine the influence of different operating conditions on flow and flame dynamics together with pollution emissions. The theoretical formulation is given in section 2 . The experimental setup and numerical methods are briefly presented in section 3. Results are discussed in section 4, and a conclusion is given in section

\section{Theoretical formulation}

In LES, each variable is decomposed into resolved and subgridded parts by a spatial filtering operation, such as $f=\bar{f}+f^{\prime \prime}$, where an over bar - denotes the spatially filtered quantities and double prime " denotes the subgrid scale quantities. The resolved parts are related to the large scale motion of turbulent flow and contain the vast majority of turbulence energy, which can be directly resolved, while the dissipative subgrid scale structures are resolved by simulation. Favre filtering is often employed in consideration of compressible flow. The Favre filtered variables can be defined as $\widetilde{f}=\overline{\rho f} / \bar{\rho}$, where the tilde $\widetilde{f}$ denotes the Favre-averaged variables. The governing equations of unsteady, reacting, multi-species turbulent flow are described below by employing a Favre-averaged filter:

$$
\frac{\partial \bar{\rho}}{\partial t}+\frac{\partial \bar{\rho} \tilde{u}_{j}}{\partial x_{j}}=0
$$




$$
\frac{\partial \bar{\rho} \tilde{u}_{i}}{\partial t}+\frac{\partial}{\partial x_{j}}\left(\bar{\rho} \tilde{u}_{i} \tilde{u}_{j}-\overline{\tau_{i j}}+\tau_{i j}^{s g s}\right)=0
$$

$$
\frac{\partial \bar{\rho} \tilde{E}}{\partial t}+\frac{\partial}{\partial x_{j}}\left(\bar{\rho} \tilde{E} \tilde{u}_{j}+\overline{q_{j}}-\tilde{u}_{i} \overline{\tau_{i j}}+H_{i}^{s g s}-\sigma_{i}^{s g s}\right)=\overline{\dot{Q}^{c}}
$$

$$
\frac{\partial \bar{\rho} \tilde{Y_{m}}}{\partial t}+\frac{\partial}{\partial x_{j}}\left(\bar{\rho} \tilde{u}_{j} \tilde{Y}_{m}+\bar{\rho} \tilde{V_{j, m}} \tilde{Y_{m}}+\phi_{i, m}^{s g s}-\theta_{i, m}^{s g s}\right)=\overline{{\overline{\rho_{m}}}^{c}},
$$

$N$. In the above equations, the superscript ${ }^{s g s}$ denotes the unsolved subgrid terms that have to be modelled for equations to be solved. The subgrid-scale stress tensor is closed by the eddy viscosity hypothesis as:

$$
\tau_{i j}^{s g s}=-2 \bar{\rho} \nu_{t}\left(\tilde{S}_{i j}-\frac{1}{3} \tilde{S}_{k k} \delta_{i j}\right)+\frac{2}{3} \bar{\rho} k^{s g s} \delta_{i j},
$$

where $\nu_{t}$ is given by $\nu_{t}=C_{\nu} k^{s g s 1 / 2} \bar{\Delta}$, and the strain rate is defined as $\tilde{S}_{i j}=$ $1 / 2\left(\partial \tilde{u}_{i} / \partial x_{j}+\partial \tilde{u}_{j} / \partial x_{i}\right)$. To obtain $\nu_{t}$, the subgrid kinetic energy $k^{s g s}$ is resolved by the following governing equation, as proposed by Menon ${ }^{15}$ :

$$
\frac{\partial \bar{\rho} k^{s g s}}{\partial t}+\frac{\partial \bar{\rho} \tilde{u}_{j} k^{s g s}}{\partial x_{j}}=P^{s g s}-D^{s g s}+\frac{\partial}{\partial x_{j}}\left(\bar{\rho} \frac{\nu_{t}}{\operatorname{Pr}_{t}} \frac{\partial k^{s g s}}{\partial x_{j}}\right)
$$

where the production term $P^{s g s}$ and the dissipation rate term $D^{s g s}$ are closed by $-\tau_{i j}^{s g s} \partial \tilde{u}_{i} / \partial x_{j}, C_{\varepsilon} \bar{\rho} k^{s g s 3 / 2} / \bar{\Delta}$ respectively. The model constants $C_{v}$ and $C_{\varepsilon}$ are chosen to be 0.067 and 0.916 , respectively, according to Calhoon ${ }^{16}$ .The remaining unclosed subgrid terms are modelled by gradient assumption method. The filtered viscous stress tensor is closed by:

$$
\tau_{i j}=\mu\left(\partial u_{i} / \partial x_{j}+\partial u_{j} / \partial x_{i}\right)-\frac{2}{3} \mu\left(\partial u_{k} / \partial x_{k}\right) \delta_{i j},
$$

The filtered diffusion velocities in the filtered governing equations of the turbulent flow are obtained using Fick's law $V_{j, m}=\left(-D_{m} / Y_{m}\right)\left(\partial Y_{m} / \partial x_{j}\right)$. The reaction source term in the energy equation is given by:

$$
\overline{\dot{Q}^{c}}=\sum_{r}\left[\sum_{m}\left(a_{m r}-b_{m r}\right)\left(\Delta h_{f}^{0}\right)_{m}\right] \dot{\omega}_{r}
$$


Pressure is determined by the equation of state for a perfect gas mixture. The detail about the numerical scheme could refer to Zheng ${ }^{17}$.

The eddy dissipation concept (EDC) model could reflect the interaction between turbulence and chemistry with low computational expense ${ }^{18}$.Thus, this model is employed in the present work to describe the flame chemistry in partially premixed conditions. The EDC combustion model was based on the assumptions that the filtered flow field is composed of surroundings $(0)$ and fine structures $(*)$ and that dissipation of turbulent kinetic energy as well as chemical reactions only take place in fine structures. The volume fraction of fine structure in each cell $\gamma^{*}$ and the mean residence time of reactive mixture within the fine structure $\tau^{*}$ are obtained by:

$$
\gamma^{*}=\operatorname{Re}_{\Delta}^{-3 / 4},
$$

following Fureby's approach ${ }^{19}$ based on energy cascade model, where $\mathrm{Re}_{\Delta}=$ $u^{\prime} \Delta / \nu$ is the filtered scale Reynolds number. The fluctuating velocity is approximated by $u^{\prime}=\sqrt{2 k^{s g s} / 3}$. The reaction term in $m$ th species conservation equation is described as:

$$
\tau^{*}=1.23 \sqrt{\frac{\Delta \mu}{\bar{\rho}\left(k^{s g s}\right)^{1.5}}},
$$

$$
\frac{\bar{\rho}\left(Y_{m}^{*}-Y_{m}^{0}\right)}{\tau^{*}}=\dot{\omega}_{m}\left(\bar{\rho}, Y_{m}^{*}\right)
$$

$Y_{m}^{0}$ and $Y_{m}^{*}$ represent $m$ th species mass fraction in the surroundings and fine structures, As each filtered spices mass fraction can be described as:

$$
\tilde{Y}_{m}=\gamma^{*} Y_{m}^{*}+\left(1-\gamma^{*}\right) Y_{m}^{0}
$$

thus, the following equation can be derived:

$$
\bar{\rho}\left(Y_{m}^{*}-\tilde{Y}_{m}\right)=\left(1-\gamma^{*}\right) \tau^{*} \dot{\omega}_{m}\left(\bar{\rho}, Y_{m}^{*}\right)
$$

In the present study, the fine structure volume fraction $\gamma^{*}$ is so small compared to the cell size that it could be set to zero, implying that the reaction takes place in a minimal region of each cell. 


\section{Experimental and numerical setup}

The schematic diagram of the experimental combustor is shown in Fig.1. The combustor consists of a 52-degree, eight spiral swirl-vaned air inlet with a swirl number 0.9 and five column fuel injectors located downstream of the vanes, followed by an annular chamber. Air and fuel are injected in opposite directions and premixed inside a short passage before combustion takes place in the chamber.

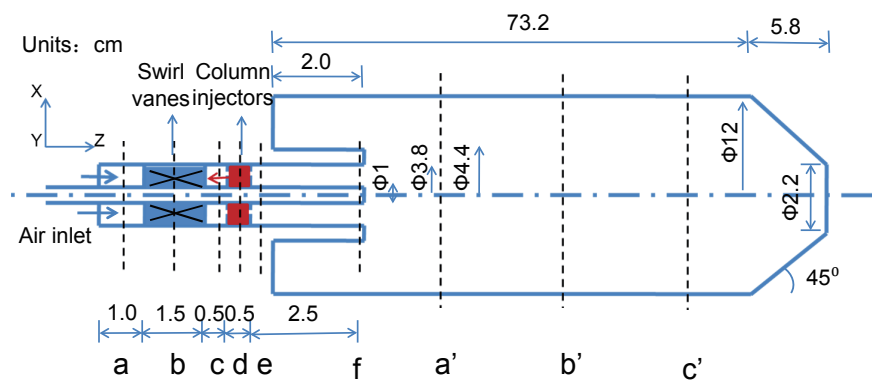

Figure 1: Schematic diagram of experimental combustor.

An empirical three-step reduced methane reaction mechanism is employed in the present study ${ }^{20}$, which is obtained by a least-squares regression empirical fitting method. Details are shown in Tab.1. Experiments conducted by Griebel $^{21}$ are used to validate the reduced reaction mechanism. Comparative results of the flame front and axial velocity field between the numerical simulation conducted by FLUENT and experiment are shown in Fig.2. Good agreement is obtained. Besides, comparison of the computational results of the outlet temperature at different equivalence ratios between the two chemical mechanisms using COSLAB with the laminar free-flame propagation model indicates that the reduced mechanism shows good correlation with the Berkeley's GRI-mech 3.0 methane chemical mechanism. These results are shown in Fig.3, which demonstrates that the three-step reduced mechanism can be applied with reasonable accuracy to the study of natural gas combustion in our cases.

A five-step NO global mechanism for a lean premixed combustor is employed to investigate $\mathrm{NO}_{\mathrm{X}}$ emissions under different operating conditions ${ }^{20}$ .The flame NO formation by Zeldovich as well as nitrous oxide mechanisms are described in step 1, while step 2 shows the prompt and NNH mechanisms. According to the analysis focusing on the relative importance of each step on $\mathrm{NO}_{\mathrm{X}}$ formation using COSILAB conducted by Zheng ${ }^{17}$, step 1 and 2 
contribute more than $50 \%$ of total $\mathrm{NO}_{\mathrm{X}}$ emission, which means that reducing the reaction rates in steps 1 and 2 is crucial to control $\mathrm{NO}_{\mathrm{X}}$ formation. The thermal NO formation rate through $\mathrm{H}$-atom and O-atom attack on $\mathrm{N}_{2} \mathrm{O}$ mechanisms is shown in steps 3 and 4, respectively. A small portion of flame NO emission through the prompt and NNH mechanisms just downstream of the flame is represented in step 5. Details are shown in Tab.2. Comparative results of $\mathrm{NO}$ emissions between the numerical simulation using COSLAB and experimental measurements ${ }^{21}$ are shown in Fig.4, which demonstrate that the reduced mechanism has reasonable accuracy.

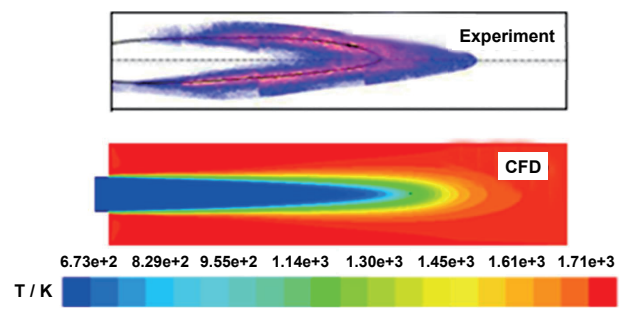

(a) comparison of flame front

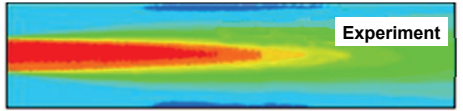

CFD $/ \mathrm{m} / \mathrm{s}$

(b) comparison of axial velocity field

Figure 2: Comparative results of the flame front and axial velocity between the experiment and numerical simulation with reduced mechanism.
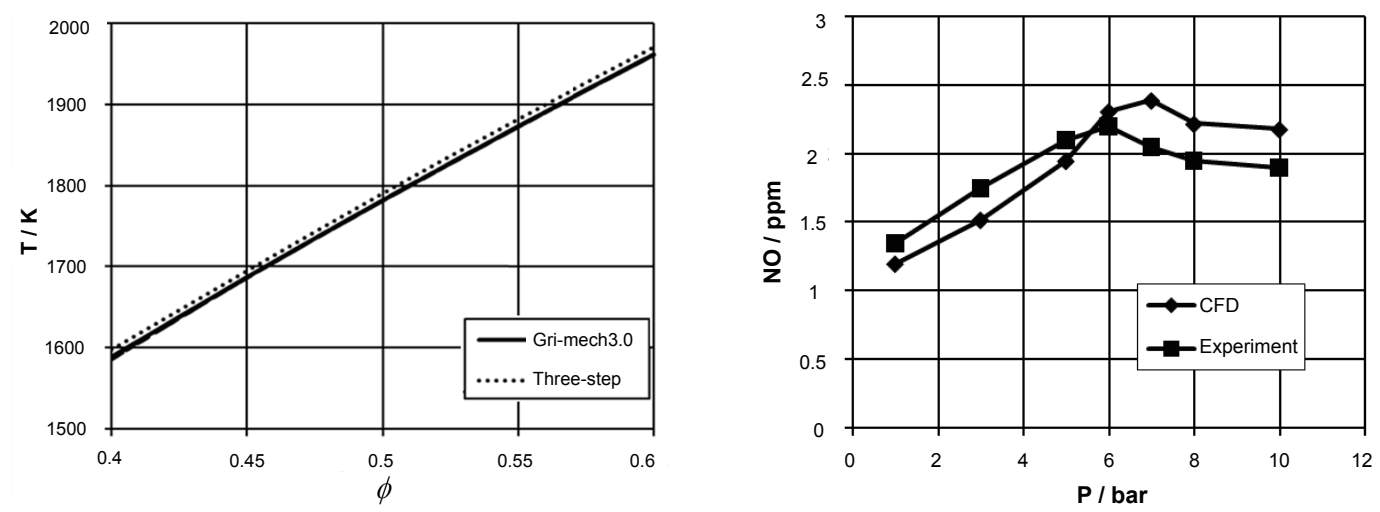

Figure 3: Comparison of the outlet temper- Figure 4: Comparative results of outlet NO ature between three-step reduced methane emissions between experiment and numerical mechanism and GRI-mech 3.0 reaction mech- simulation with five-step NO global mechaanism.

nism.

Table 1: Three step global mechanism for methane 


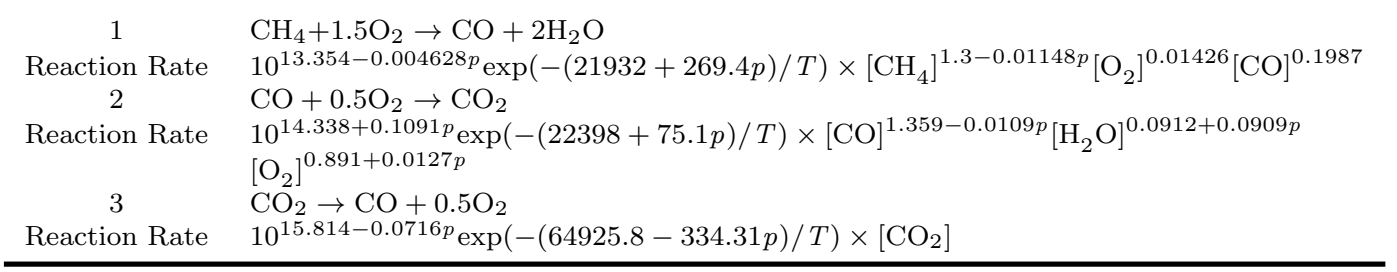

Table 2: Five step global mechanism for NO formation

\begin{tabular}{clc}
\hline Step & \multicolumn{1}{c}{ Equation } & Mechanism \\
\hline 1 & $\mathrm{~N}_{2}+\mathrm{O}_{2} \Rightarrow 2 \mathrm{NO}$ & Zeldovich and nitrous oxide mechanisms \\
Reaction Rate & $10^{14.122+0.0376 p} \exp \{-(46748+126.6 p) / T\} \times[\mathrm{CO}]^{0.8888-0.0006 p}\left[\mathrm{O}_{2}\right]^{1.1805+0.0344 p}$ \\
2 & $\mathrm{~N}_{2}+\mathrm{O}_{2} \Rightarrow 2 \mathrm{NO}$ & prompt and NNH mechanisms \\
Reaction Rate & $10^{29.8327}-4.7822 \log p \exp \{-(61265+704.7 p) / T\} \times[\mathrm{CO}]^{2.7911-0.048 p}\left[\mathrm{O}_{2}\right]^{2.4613}$ \\
3 & $\mathrm{~N}_{2}+\mathrm{O}_{2} \Rightarrow 2 \mathrm{NO}$ & $\mathrm{H}-$ atom attack on $2 \mathrm{O}$ mechanism \\
Reaction Rate & $10^{14.592} \exp (-69158 / T)\left[\mathrm{N}_{2}\right]\left[\mathrm{H}_{2} \mathrm{O}\right]^{0.5} \times\left[\mathrm{O}_{2}\right]^{0.25} T^{-0.7}$ \\
4 & $\mathrm{~N}_{2}+\mathrm{O}_{2} \Rightarrow 2 \mathrm{NO} \quad$ O-atom attack on N2O mechanism \\
Reaction Rate & $10^{10.317} \exp (-52861 / T) \times\left[\mathrm{N}_{2}\right]\left[\mathrm{O}_{2}\right]$ & prompt and NNH mechanisms \\
5 & $\mathrm{~N}_{2}+\mathrm{O}_{2} \Rightarrow 2 \mathrm{NO}$ & \\
Reaction Rate & $10^{14.967} \exp (-68899 / T) \times\left[\mathrm{N}_{2}\right]\left[\mathrm{O}_{2}\right]^{0.5} T^{-0.5}$ \\
\hline
\end{tabular}

The computational fluid dynamics modelling program is based on KIVA-4 code, in which the Arbitrary Lagrangian Eulerian (ALE) numerical scheme is employed based on the finite volume method. Each cycle is divided into three phases. In phases 1 and 2, the grid vertices move with the fluid velocity, and convection is absent across cell boundaries; the Lagrangian calculation is then performed. Mass and energy source terms resulting from the chemical reaction are computed in phase 1, while diffusion terms and other source terms in the governing equations are obtained in phase 2 using a modified SIMPLE (Semi-Implicit Method for Pressure-Linked Equation) method. In phase 3, the flow field is rezoned onto the new computational mesh and then frozen, and convection terms are calculated by means of a second-order accuracy quasi-second-order upwind scheme based on an explicit method. Courant stability restrictions are absent as Lagrangian computation is largely implicit and Eulerian calculation can be subcycled. According to code validation and grid dependence test conducted by Zheng ${ }^{17}, 1.3$ million cells in an unstructured mesh with a grid resolution of $0.1 \mathrm{~cm}$ is applied in the present work. Velocity-inlet and outflow conditions are used along with no-slip, adiabatic walls. The platform with Intel Nehalem $2.26 \mathrm{GHz}$ CPU in Lancaster University and the HPC platform with Intel Xeon 5670 in Tsinghua University are used to complete the computation. 


\section{Results and discussion}

\subsection{Non-reacting cases}

Contour plots of the vorticity field corresponding to different cross-sections from axial locations $a$ to $f$ marked in Fig.1 are shown in Fig.5. A confined high vorticity area is observed in Fig.5(a) close to the walls due to strong shear stresses caused by the velocity difference between the walls and the ambient fluid at the air inlet. As the flow and vortices shift downstream and pass through the swirl vanes, the shear and stretch rates increase rapidly due to swirl effects, which contribute to more frequent vortex production and breakdown as well as vortex shedding. The vorticity field corresponding to the swirler location is shown in Fig.5(b). Counter flow between the swirling air and injected fuel make the shear stress increase further, and as a result, more widely intense vortex distributions are observed in Fig.5(c). However, high vorticity zones deviate from the central body walls, which indicate the centrifugal force effect induced by swirl flow. Because the installation angle of the column fuel injectors are different from those of the swirl vanes, the position discrepancy effect will collaborate with the swirl effect to enhance fuel/air mixing and result in an asymmetrical distribution of vorticity. This asymmetry is shown in Fig.5(d) and becomes distinct in Fig.5(e). When the fuel/air mixture moves down to the dump plane, also referred as location $f$, the asymmetrical distribution of high vorticity has nearly vanished, and the deviation distance is further increased, which can be speculated from Fig.5(f).

Contour plots of the vorticity field and the corresponding streamlines at different axial locations $a^{\prime}$ to $c^{\prime}$ are shown in Fig.6. As the mixture moves into the chamber, the flow scale expands suddenly, and the vorticity magnitude decreases due to the vortices extending according to the conservation of angular momentum. Small portion of high vorticity distribution can be observed in Fig.6(a) at axial location $a^{\prime}$, but is less evident in Fig.6(b) and Fig.6(c). The damped tangential velocity leads to the radial pressure gradient under the effects of centrifugal force. A low-pressure core emerges and aligns with the axial axis at a downstream location next to the dump plane, which results in a concentrated distribution of spanwise vortices around the symmetry axis, which can be easily seen in Fig.6(d) and Fig.6(e). However, from the streamwise of location $c^{\prime}$ shown in Fig.6(f), the core is propelled away from the centreline and expands downstream helicoidally due to vortex core precession; spiral vortices breakdown gives rise to more stagnation 


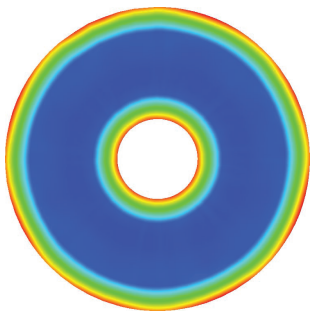

(a) $\mathrm{Z}=0.5 \mathrm{~cm}$

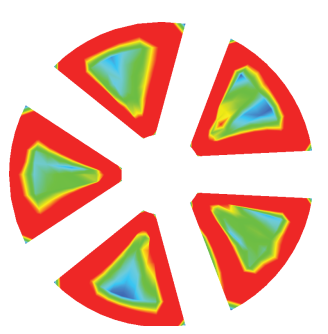

(d) $\mathrm{Z}=3.25 \mathrm{~cm}$

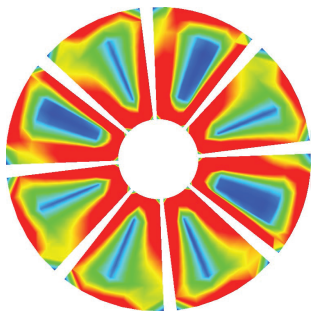

(b) $\mathrm{Z}=1.25 \mathrm{~cm}$

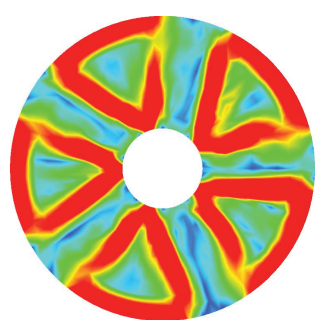

(e) $\mathrm{Z}=4 \mathrm{~cm}$

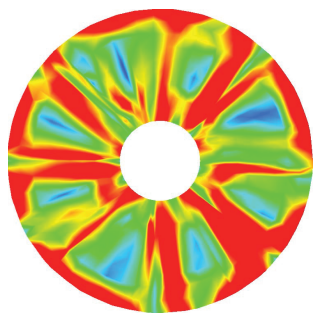

(c) $\mathrm{Z}=2.75 \mathrm{~cm}$

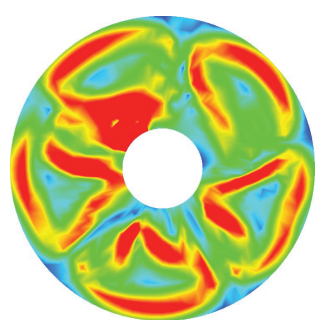

(f) $\mathrm{Z}=6 \mathrm{~cm}$

$\Omega / s$

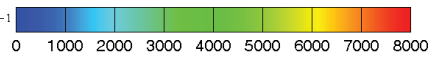

Figure 5: Contour plots of vorticity field corresponding to different axial locations.

points and vortices numbers with different sizes, which is consistent with the conclusions of Wang et al. ${ }^{22}$.As the frequency of vortex core precession increases with increased Reynolds number, many vortices with high vorticity next to the combustor walls are observed with high Reynolds number, leading to increased tangential velocity due to the squeezing effects against the wall. This can be inferred from Fig.7(c), where high velocity magnitudes appear around the chamber wall compared to Fig.7(a) and Fig.7(b).

\subsection{Reacting cases}

The following section investigates the influence of swirl number, Reynolds number, equivalence ratio and nitrogen dilution on combustion dynamics and NOx emissions under the standard operating condition of $\operatorname{Re}=6.3 \times 10^{4}$, $\varphi=0.65, p=3 b a r$ and fuel composition given by $\mathrm{CH}_{4}: \mathrm{N}_{2}=4: 1$.

\subsubsection{Swirl number effects}

In gas turbine engines, the bubble and helix modes are the most popular type of vortex breakdown due to Kelvin-Helmholtz (K-H) instability in the axial and azimuthal shear layers. Helix mode usually takes place on the outer 


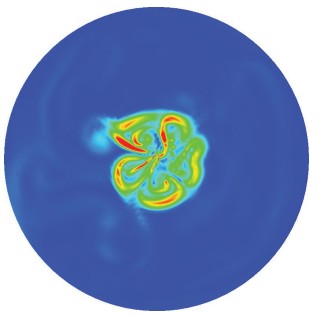

(a) $\mathrm{Z}=10 \mathrm{~cm}$

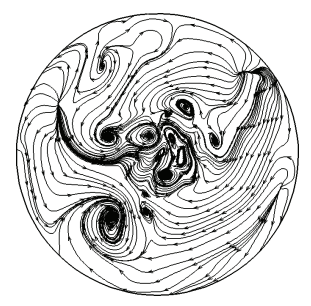

(d) $\mathrm{Z}=10 \mathrm{~cm}$

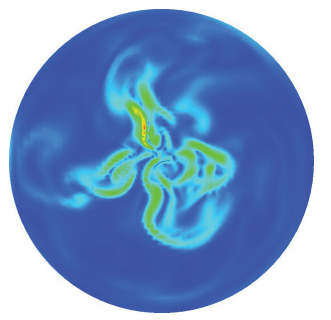

(b) $\mathrm{Z}=20 \mathrm{~cm}$

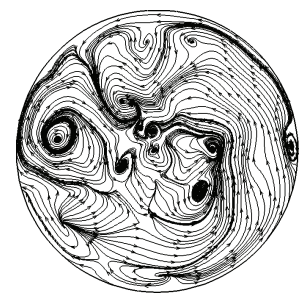

(e) $\mathrm{Z}=20 \mathrm{~cm}$

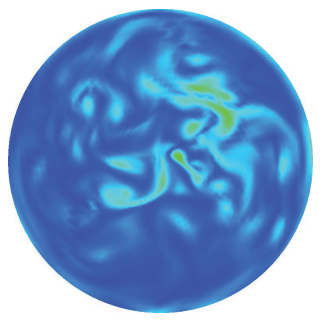

(c) $\mathrm{Z}=30 \mathrm{~cm}$

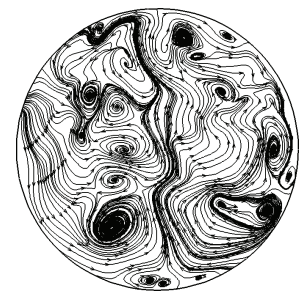

(f) $\mathrm{Z}=30 \mathrm{~cm}$

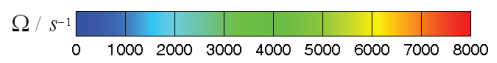

Figure 6: Contour plots of vorticity field and corresponding streamlines at different axial locations.

edge of the shear layers and behaves like large scale spiral vortices rotating in the combustor and then crushing into several fine scale vortices; bubble mode often occurs on the edge of shear layers and behaves as small scale vortices shed along the axial direction. The iso-surface of vorticity $(\Omega=5000)$ at locations of $r<0.01 \mathrm{~m}$ and $r>0.01 \mathrm{~m}$ with different swirl numbers are shown in Fig.8, where $r$ is the approximate location of the shear layer. More fine-scale vortices shed from the large-scale vortices and the initial breakdown position moves upstream with increased swirl number, which can be inferred from Fig.8(a) and Fig.8(b). Obvious small-scale spiral vortices breakdown can be observed in Fig.8(c). However, as the swirl number increases to 1.2, as shown in Fig.8(d), such phenomena become inconspicuous, which can be interpreted as the disturbance arising from bubble-type vortex breakdown developing upstream to the flame root and weakening the helix instability with an increased swirl number.

To analyse the flame regimes in a partially premixed combustion process, 


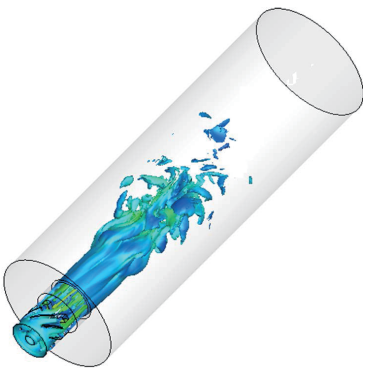

(a)

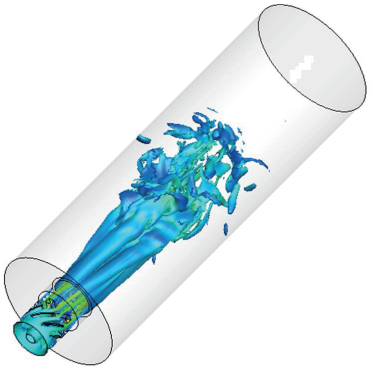

(b)

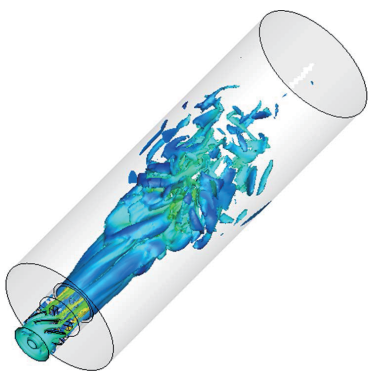

(c)

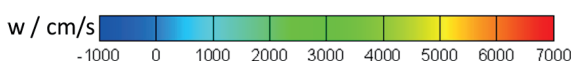

Figure 7: Iso-surfaces of vorticity field flooded with axial velocity with different Re numbers. (a) $\operatorname{Re}=5.3 \times 10^{4} ;(\mathrm{b}) \operatorname{Re}=6.3 \times 10^{4} ;(\mathrm{c}) \operatorname{Re}=7.4 \times 10^{4}$.

a modified flame index is applied as following definition:

$$
\mathrm{MFI}=\frac{\nabla Y_{\text {fuel }} \cdot \nabla Y_{\mathrm{O}_{2}}}{\left|\nabla Y_{\text {fuel }} \cdot \nabla Y_{\mathrm{O}_{2}}\right|},
$$

where $Y_{\text {fuel }}$ and $Y_{\mathrm{O}_{2}}$ represent the fuel and oxidizer mass fractions, respectively. When MFI $>0$, the combustion is occurring in a premixed regime; the diffusion regime is confirmed when MFI $<0$. The flame index distributions with different swirl numbers are shown in Fig.9. where the flame is characterized by an iso-surface with $\mathrm{T}=1800 \mathrm{~K}$. The entire flame structure is shown in the centre of the figure, while the diffusion and premixed flames are exhibited at left and right positions, respectively. Proportion of premixed combustion increases with higher swirl numbers relating to intense bubble vortex breakdown and entrainment effects in the recirculation zones. The premixed region is also shown to move upstream when the swirl number increases further, which can be explained by improved mixing between the fuel and oxidizer ahead of the combustion taking place in the upstream region. Here, the unmixedness parameter ${ }^{23}$ is introduced to identify the effect of swirl number on the mixing process. The parameter is defined as:

$$
\mathrm{U}=Y_{f}^{\prime \prime} / \overline{Y_{f}}\left(1-\overline{Y_{f}}\right),
$$

where $\overline{Y_{f}}$ and $Y_{f}^{\prime \prime}$ represent the time-averaged fuel mass fraction and its fluctuation over the mean value, respectively. A near zero value of $U$ indicates better mixing has been achieved. By collecting valid data along the flow 
field, a probability density function (PDF) of $\mathrm{U}$ is obtained:

$$
\mathrm{P}(\mathrm{U})=\sum_{i=1}^{\mathrm{N}}\left[\mathrm{H}\left(\mathrm{U}_{i}-\mathrm{U}\right)-\mathrm{H}\left(\mathrm{U}_{i}-\mathrm{U}-\Delta \mathrm{U}\right)\right] / \mathrm{N} \Delta \mathrm{U},
$$

where $\mathrm{H}$ is Heaviside function, $\Delta \mathrm{U}$ represents the selected interval of unmixedness, $\mathrm{N}=\mathrm{U} / \Delta \mathrm{U}$. The PDF of $\mathrm{U}$ at a position downstream of the swirl vanes with different swirl number is shown in Fig.10. The narrower distribution of the PDF near zero with an increased swirl number indicates better mixing between the fuel and oxidizers, which results in an increased ratio of premixed combustion along with a reduction of temperature and $\mathrm{NO}_{\mathrm{X}}$ emissions.

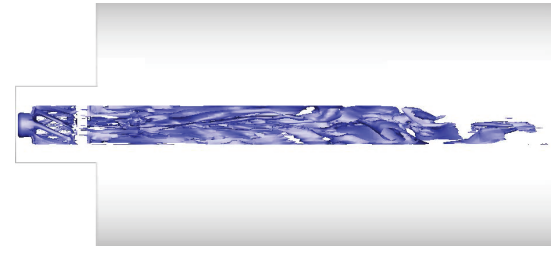

(a) $S=0.9, r<0.01 m$

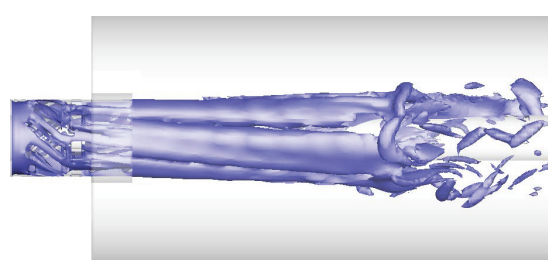

(c) $S=0.9, r>0.01 m$

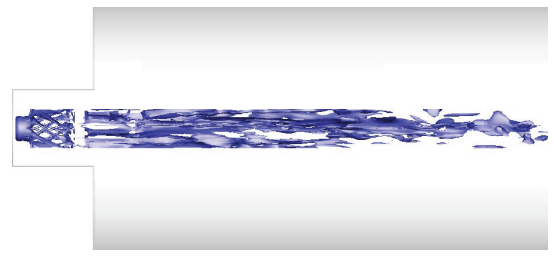

(b) $S=1.2, r<0.01 m$

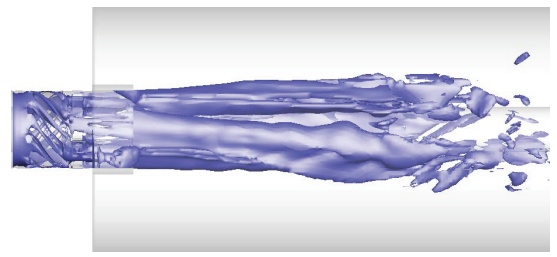

(d) $S=1.2, r>0.01 m$

Figure 8: Iso-surfaces of vorticity $(\Omega=5000)$ with different swirl numbers, $r$ is the approximate location of the shear layer.

In order to quantificationally analyse the partially premixed combustion, the proportion of premixed combustion (PPC) is defined as:

$$
\operatorname{PPC}=\frac{\int_{\text {MFI }>0} d V}{\int_{M F I} d V}
$$

Fig.11 shows the proportion of premixed combustion and $\mathrm{NO}_{\mathrm{X}}$ emissions with different swirl numbers. The proportion of premixed regime only accounts 


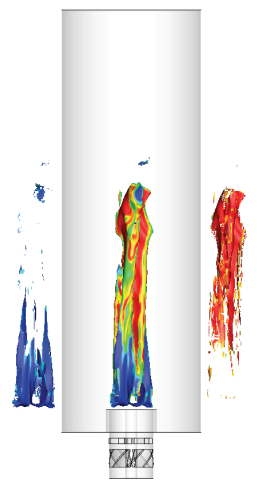

(a)

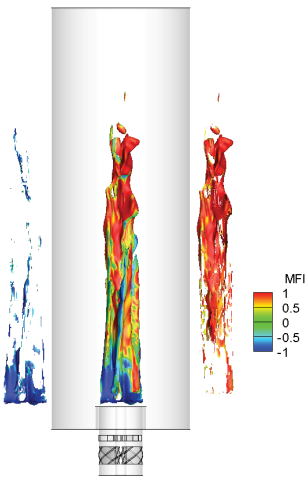

(b)

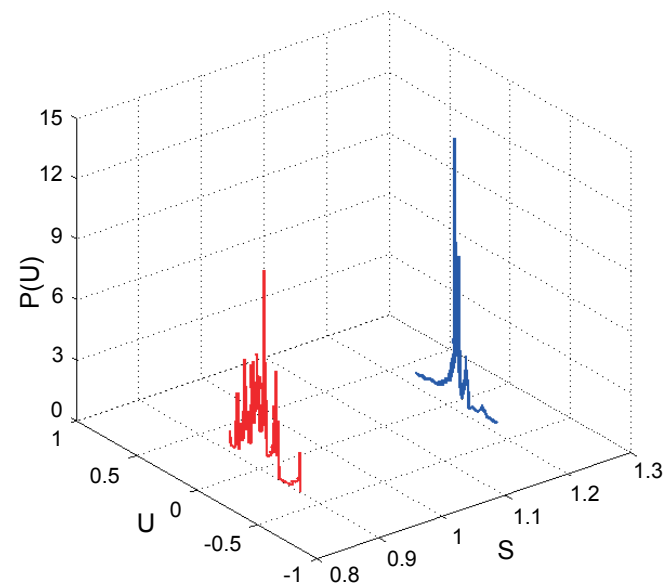

Figure 9: Modified flame index distribution with different swirl numbers.(a) $S=$ Figure 10: PDF distribution of unmixedness $0.9 ;(\mathrm{b}) S=1.2$. with different swirl numbers(axial location c).

for $20 \%$ at most during the partially premixed combustion, which indicates that partially premixed combustion occurring more like diffusion flame at lean intense swirling conditions. This is consistent with the conclusion of Meier $^{7}$. PPC increases when the swirl number increases from 0.9 to 1.2 due to better mixing between the fuel and oxidizers. In lean premixed combustion, NO formation reaction rates of step 1 and 2 reduce significantly; this normally results in low $\mathrm{NO}_{\mathrm{X}}$ emissions. However, the reduction degree of $\mathrm{NO}_{\mathrm{X}}$ formation is not so distinct compared to the increase of the swirl number, which can be interpreted as a large swirl number contributing to better mixing between the fuel and oxidizers while also leading to a long retention time of reaction products arising from intense entrainment, which facilitates the formation of NO. Thus, an appropriate swirl number is critical for gas turbine chamber design to achieve superior mixing and minimal pollution emissions.

For the purpose of studying the frequency and amplitude of instability during combustion with the Fourier spectra analysis method, several monitoring points are set along the flow field to record the time variation of the thermal variables. A typical point located in the shear layer at midstream $\mathrm{Z}=15 \mathrm{~cm}$ is chosen for the sake of capturing both shear layer instability and vortex breakdown instability. A fast Fourier transform (FFT) of the temperature trace for different swirl numbers is shown in Fig.12, where the dominant frequency indicates shear layer instability and harmonic waves indicate vor- 
tex breakdown instability. The swirl number does not show much influence on the dominant frequency, and all flames' temperature experience a dominant frequency of approximately $995 \mathrm{~Hz}$, as derived from the shear layer instability. However, the amplitude corresponding to the main frequency is shown to increase with increasing swirl number, which can be explained as an intense velocity fluctuation with increased swirl number. More harmonic waves also show that vortex breakdown instability appears when the swirl number increases.

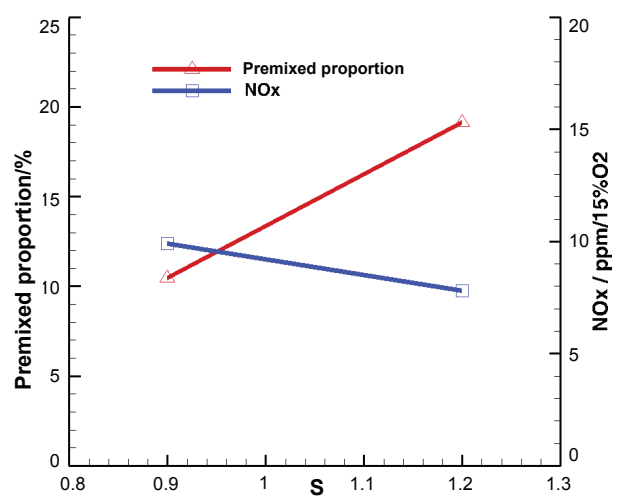

Figure 11: Proportion of premixed combustion and $\mathrm{NO}_{\mathrm{X}}$ emission with different swirl Figure 12: FFT analysis of temperature with numbers.

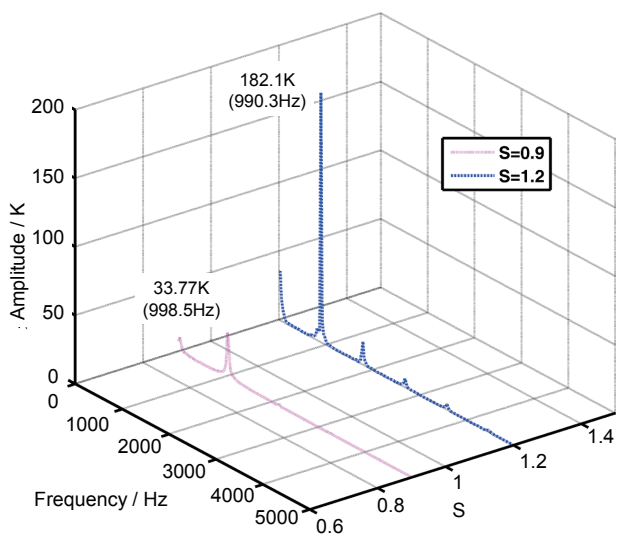

\subsubsection{Reynolds number effects}

The CRZs characterized by the dotted lines with different Reynolds numbers in the reacting cases are shown in Fig.13. The size of the CRZ is shown to increase due to larger pressure drop induced by increased axial momentum, and the vortex structures become more symmetrical with increased Reynolds numbers; this is consistent with the observation in non-reacting cases. However, the size increases beyond that of corresponding cases without reactions under the same Reynolds number due to thermal expansion effects arising from the chemical reaction. A larger low-speed region appears at larger Reynolds number, which improves flame stability during partially premixed combustion.

From the analysis of non-reacting cases, high Reynolds numbers contribute to more frequent vortex breakdown and consequently better degrees of mixing between the fuel and oxidizers. Fig.14 shows the PDF distribution 
of the unmixedness parameter just downstream of the swirl vanes with different Reynolds numbers. The better degree of mixing between the fuel and oxidizers can be concluded from the narrower distribution of the PDF near zero with increased Reynolds number. Due to the better mixing between the fuel and oxidizers, the length of the diffusion flame is decreased while the premixed flame is slightly increased with increased Reynolds numbers; this can be seen in the modified flame index distribution shown in Fig.15, where the flame surface is still characterized by an iso-surface of temperature $\mathrm{T}=1800 \mathrm{~K}$. Moreover, being different from the effect of the swirl number on $\mathrm{NO}_{\mathrm{X}}$ formation, large Reynolds numbers shorten the retention time of hot reaction products, which indicates that $\mathrm{NO}_{\mathrm{X}}$ emissions can be suppressed effectively. As the frequency and amplitude of the shear layer and vortex breakdown instabilities increase with Reynolds number, the entire flame becomes more wrinkled, and necking effects can be seen with the largest Reynolds number case shown in Fig.15(c).

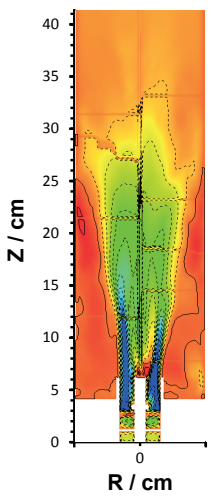

(a)

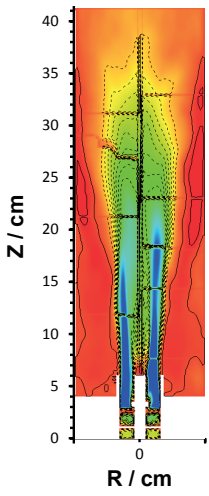

(b)

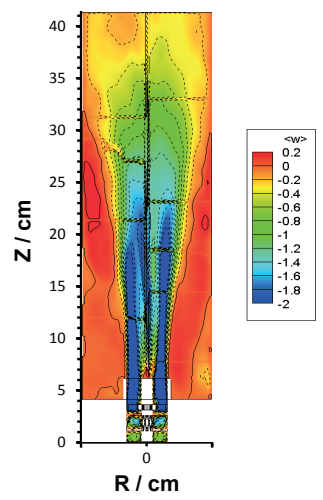

(c)

Figure 13: The CRZs characterized by the dotted lines with different Re numbers.(a)Re $=$ $5.3 \times 10^{4} ;(\mathrm{b}) \operatorname{Re}=6.3 \times 10^{4} ;(\mathrm{c}) \operatorname{Re}=7.4 \times 10^{4}$.

\subsubsection{Equivalence ratio effects}

Lean premixed combustion appears to be a promising technology for pollution reduction in gas turbine engines. In the present study, lean premixed combustion is achieved by adjusting the inlet flow rate. The contour plots of the time-averaged temperature with different equivalence ratios at $\operatorname{Re}=6.3 \times 10^{4}, p=3$ bar and composition given by $\mathrm{CH}_{4}: \mathrm{N}_{2}=4: 1$ are 


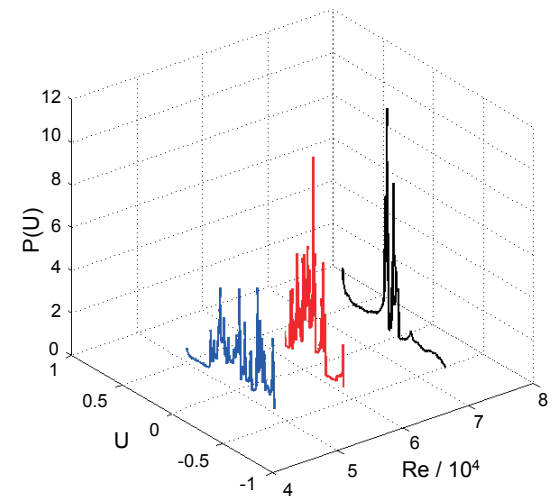

Figure 14: PDF distribution of unmixedness with different Re numbers(axial location c).

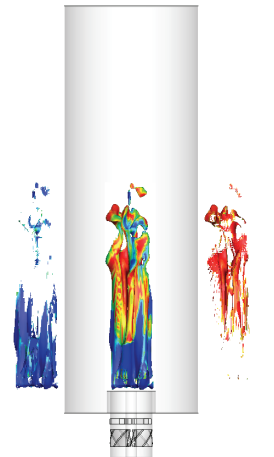

(a)

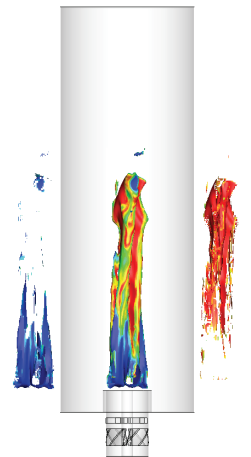

(b)

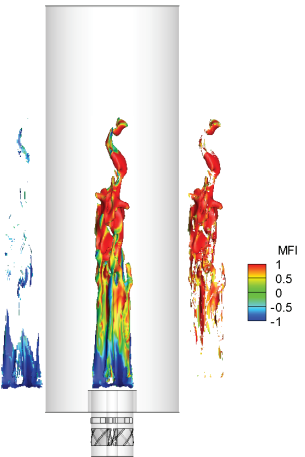

(c)

Figure 15: Modified flame index distribution with different Re numbers.(a)Re $=5.3 \times$ $10^{4} ;(\mathrm{b}) \operatorname{Re}=6.3 \times 10^{4} ;(\mathrm{c}) \operatorname{Re}=7.4 \times 10^{4}$.

shown in Fig.16. When the equivalence ratio approaches the lean flammability of 0.45 for methane combustion, the high temperature region and flame length dramatically decrease due to the chemical reaction of fuel and excess air, which is crucial for emission reductions of thermal $\mathrm{NO}_{\mathrm{X}}$. However, lean combustion tends to motivate combustion instabilities, such as flame flashback or blow off. From the instantaneous temperature distribution along the centreline shown in Fig.17, large fluctuations of temperature emerge at an equivalence ratio of 0.5 near the flame root at approximately $Z=10 \mathrm{~cm}$ compared to other cases. A decrease of equivalence ratio is consistent with a decrease in the velocity of fuel injection, and due to the counter flow directions of the fuel and swirl air, the shear stress and concentration gradient 
is also decreased. Mixing is thus reduced compared to high ratio cases. Premixed combustion proportions during partially premixed combustion process significantly reduce when the equivalence ratio approaches the lean flammability of 0.45 for methane combustion. When the mixture flows into the chamber and ignites, many local positions are still at the equivalence ratio below the lean flammability or the combustion reaction does not take place. Such a phenomenon leads to temperature nonuniformity near the flame root.

Fig.18 shows the proportion of premixed combustion and $\mathrm{NO}_{\mathrm{X}}$ emissions at different equivalence ratios. According to the above analysis, a smaller equivalence ratio lowers the proportion of premixed combustion but also reduces $\mathrm{NO}_{\mathrm{X}}$ formation. Fig.19 shows the iso-surfaces of NO mass fractions at different equivalence ratios, in which active regions of NO formation move upstream with decreased equivalence ratios, which give rise to shorter reaction zones and relatively small high temperature flame surfaces. Consequently, thermal $\mathrm{NO}_{\mathrm{X}}$ reduces significantly. The iso-surface of $Y_{N O}=1 \times 10^{-6}$ shown in Fig.19(c) has also entered into the vortex breakdown region downstream of the chamber, and intense turbulent pulsation along with stirring effects will enhance $\mathrm{NO}_{\mathrm{X}}$ formation.

The FFT of the flame temperature trace at different equivalence ratios is shown in Fig.20. The main frequency and amplitude of the shear layer instability vary unapparently when the equivalence ratio reduces from 0.8 to 0.65 ; harmonic waves corresponding to vortex breakdown instability also shows the same tendency. However, as the ratio approaches the lean flammability, the main frequency and amplitude of the shear layer instability increase suddenly, and the fluctuation of temperature increases from $33.45 \mathrm{~K}$ to 80.01 K. Many harmonic waves appear around the main frequency, indicating the complexity of the flame instability at lean ratio conditions. 


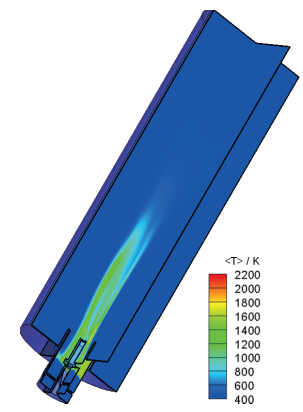

(a)

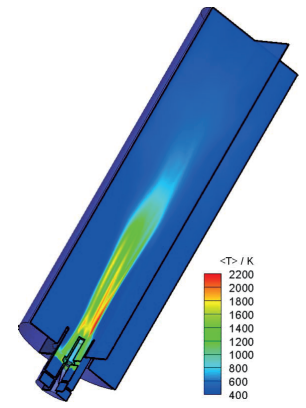

(b)

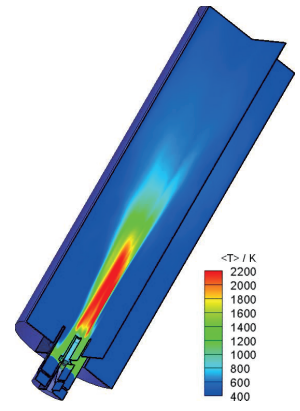

(c)

Figure 16: Contour plots of time-averaged temperature at different equivalence ratios. (a) $\varphi=0.5 ;(\mathrm{b}) \varphi=0.65 ;(\mathrm{c}) \varphi=0.8$.
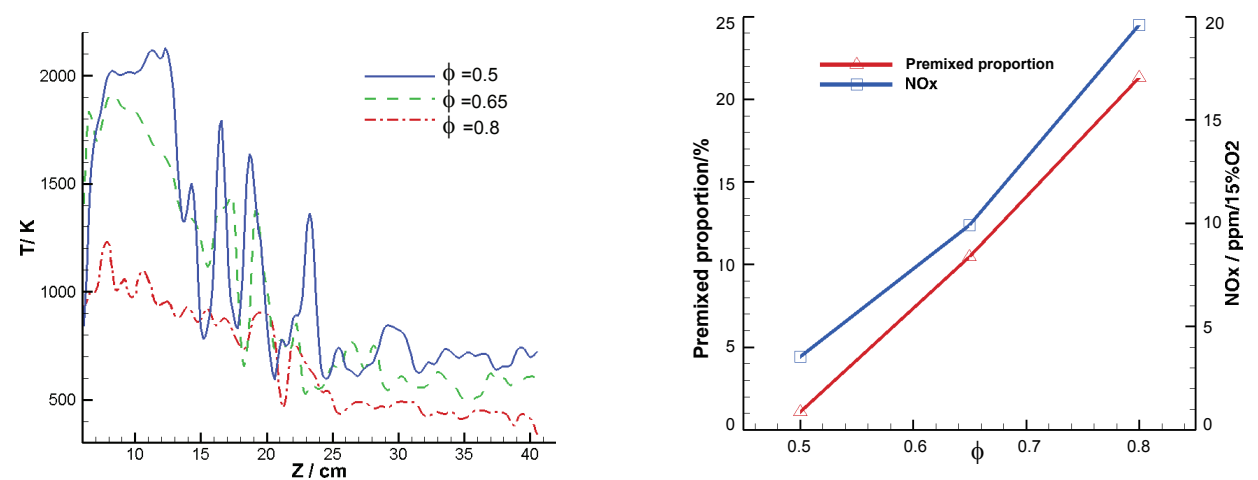

Figure 18: Proportion of premixed combusFigure 17: Instantaneous temperature along tion and $\mathrm{NO}_{\mathrm{X}}$ emissions with different equivthe centerline at different equivalence ratios. alence ratios.

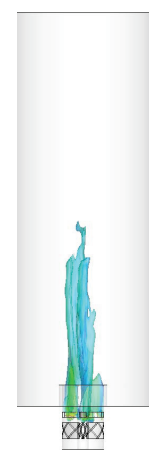

(a)

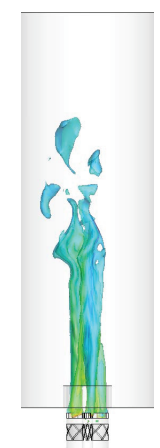

(b)

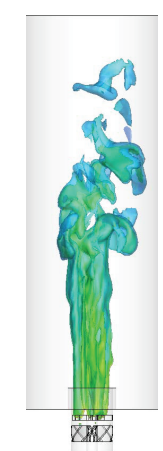

(c)

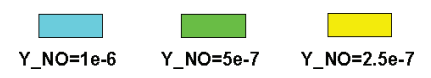

Figure 19: Iso-surface of NO mass fraction with different equivalence ratios.(a) $\varphi=$ $0.5 ;(\mathrm{b}) \varphi=0.65 ;(\mathrm{c}) \varphi=0.8$. 


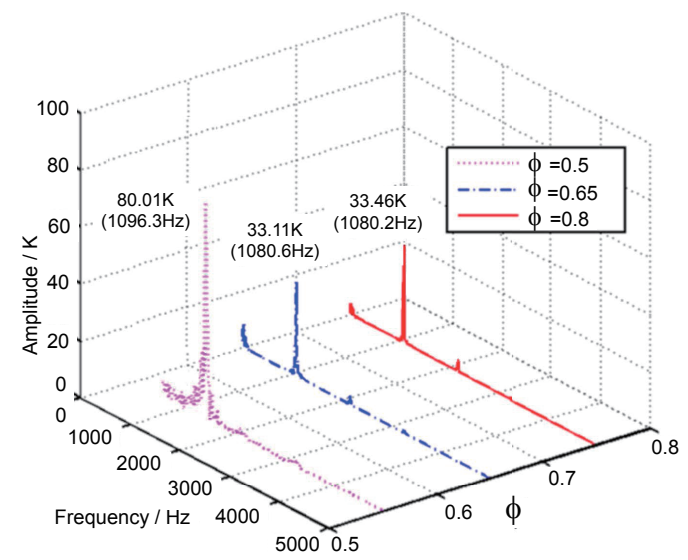

Figure 20: FFT analysis of temperature at different equivalence ratios.

\subsubsection{Nitrogen dilution effect}

During gas turbine combustion processes, inert gas is usually applied to dilute the natural gas to reduce pollution emissions. In the present work, nitrogen gas is used to form three mixed gases $\left(\mathrm{CH}_{4}: \mathrm{N}_{2}=1: 0,4: 1,2: 1\right)$, which are then examined to analyse the effect of nitrogen dilution on combustion dynamics. The time-averaged temperature and flame length are reduced with the increased proportion of $\mathrm{N}_{2}$, as shown in Fig.21. The flame temperature is decreased proportionally upstream of combustor with an increased nitrogen volume fraction and rather than a uniform temperature distribution being obtained downstream; this indicates that nitrogen dilution can dramatically reduce thermal $\mathrm{NO}_{\mathrm{x}}$ emissions.

Due to the effects of $\mathrm{N}_{2}$ dilution, heat release is decreased compared to pure methane gas and consequently the central recirculation zone is reduced due to lower thermal expansion. The high molecular weight of $\mathrm{N}_{2}$ will tend to weaken the swirl flow and azimuthal momentum, which results in a lower frequency of vortex breakdown. Such phenomena can be inferred from Fig.22, which shows fewer spanwise vortices. Because poor mixing is achieved with an increased $\mathrm{N}_{2}$ percentage, the premixed combustion regime is decreased during the operating process, but lower thermal $\mathrm{NO}_{\mathrm{x}}$ emissions are obtained due to a lower flame temperature and smaller reaction zones. The proportion of the premixed combustion regime and thermal $\mathrm{NO}_{\mathrm{x}}$ emissions with different $\mathrm{N}_{2}$ content is shown in Fig.23.

The FFT of the flame temperature trace with different nitrogen contents is shown in Fig.24. The fluctuation of temperature decreases from $33.11 \mathrm{~K}$ 
to $16.41 \mathrm{~K}$ with increased $\mathrm{N}_{2}$ content, which can be explained by the weakened effect of swirl movement caused by the larger molecular weight of $\mathrm{N}_{2}$. However, the amplitude increases to $62.57 \mathrm{~K}$ when the $\mathrm{N}_{2}$ content content is further increased, and the dilution effects make the mixture approach the lean flammability of methane combustion. As a result, combustion instability arises and becomes more severe when the amount of inert gas further increases.

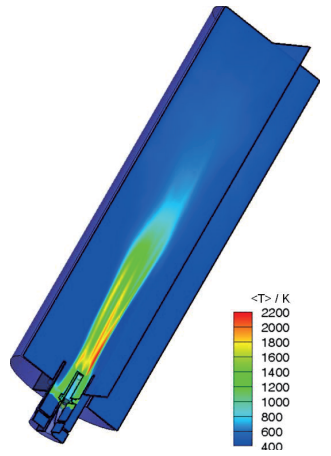

(a)

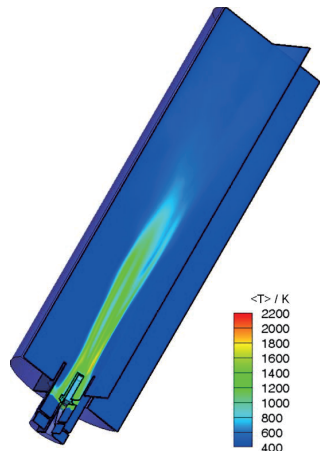

(b)

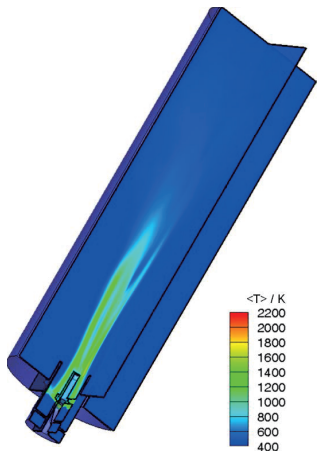

(c)

Figure 21: Contour plots of time-averaged temperature with different $\mathrm{N}_{2}$ contents.(a) $\mathrm{CH}_{4}: \mathrm{N}_{2}=1: 0 ;$ (b) $\mathrm{CH}_{4}: \mathrm{N}_{2}=4: 1 ;$ (c) $\mathrm{CH}_{4}: \mathrm{N}_{2}=2: 1$.

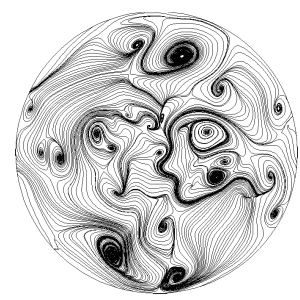

(a)

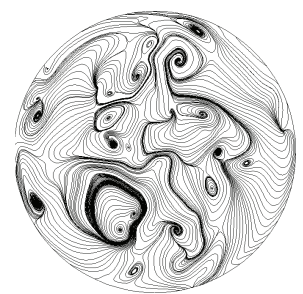

(b)

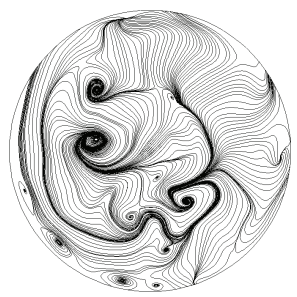

(c)

Figure 22: Spanwise vortices structure with different $\mathrm{N}_{2}$ contents.(a) $\mathrm{CH}_{4}: \mathrm{N}_{2}=1: 0 ;$ (b) $\mathrm{CH}_{4}: \mathrm{N}_{2}=4: 1 ;\left(\right.$ c) $\mathrm{CH}_{4}: \mathrm{N}_{2}=2: 1$.

\section{Conclusion}

An experimental lean partially premixed swirling combustor is studied using the large-eddy simulation method for both non-reacting and reacting 

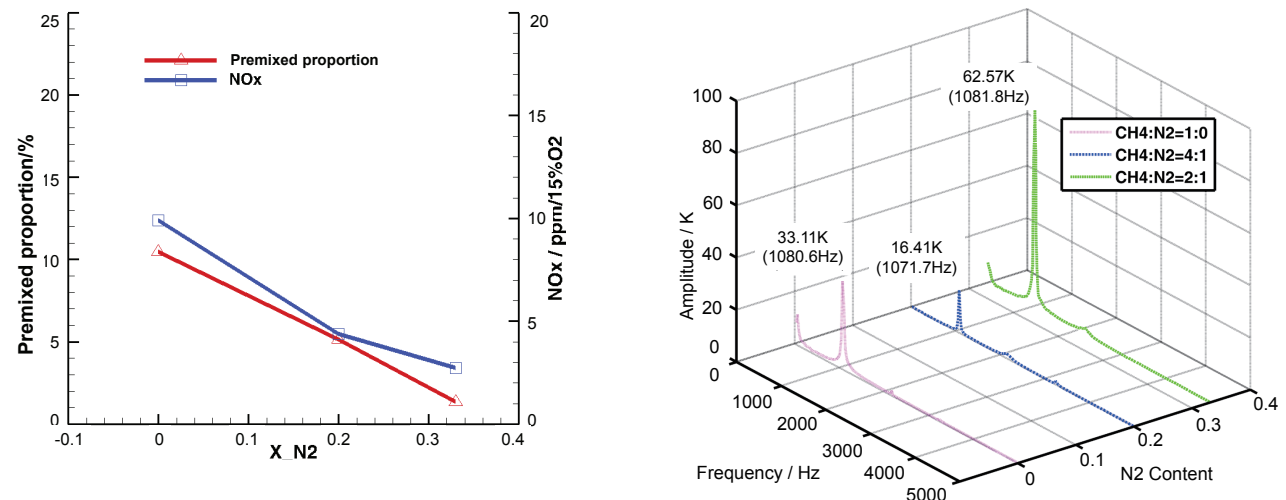

Figure 23: The proportion of premixed combustion regime and thermal $\mathrm{NO}_{\mathrm{X}}$ emissions Figure 24: FFT analysis of temperature with with different $\mathrm{N}_{2}$ contents. different $\mathrm{N}_{2}$ contents.

cases under different operating conditions. In the non-reacting cases, the intrinsic effects of swirl vanes and counter flow on flow dynamics are investigated by analysing the vorticity magnitude and streamlines at different axial positions. The influence of Reynolds number on vortex structures and vortex breakdown shows that a high Reynolds number contributes to more frequent vortex breakdown and the appearance of high vorticity near the combustor walls.

In the reacting cases, the influence of swirl number, Reynolds number, equivalence ratio and nitrogen dilution on combustion dynamics and $\mathrm{NO}_{\mathrm{X}}$ emissions are examined. The conversion between bubble- and helix-type vortex breakdown is shown to be directly related to the swirl number. The proportion of the premixed flame regime is shown to increase with swirl number and with shear layer instability and vortex breakdown instability. The length of the diffusion flame is shown to decrease while the premixed flame is slightly increased with increased Reynolds number. As the equivalence ratio approaches the lean flammability, the main frequency and amplitude of the shear layer instability increased suddenly, and the fluctuation of temperature increases from $33.45 \mathrm{~K}$ to $80.01 \mathrm{~K}$. Many harmonic waves are also shown to appear near the main frequency. Flame instability at a lean ratio is very complex. The flame lengths and heat release are shown to decrease with increased $\mathrm{N}_{2}$ content. Due to the larger molecular weight of $\mathrm{N}_{2}$, additional inert gas tends to restrain the swirl flow, which decreases the size of recirculation zones and the frequency of vortex breakdown. The proportion of the premixed flame regime and $\mathrm{NO}_{\mathrm{X}}$ emissions are also reduced with higher $\mathrm{N}_{2}$ 
content. But the combustion instability becomes severe with the increased amount of inert gas.

As the future design of gas turbine combustor is driven by higher powerdensities, lower $\mathrm{NO}_{\mathrm{X}}$ emissions, and excellent combustion stability, lean premixed (LP) combustion can prove to be a promising technology for reducing pollutant emissions and improving fuel efficiency. The conclusions obtained for flame dynamics and instability mechanism under lean partially premixed combustion regime with intense swirl flow would help to provide essential information for the design of gas turbine combustor systems, as most practical combustors operate with lean partially premixed mode of combustion.

\section{Acknowledgements}

This work is supported by the UK EPSRC through Grant EP/K036750/1 and the National Natured Science Foundation of China through Grant No. 51376107. The computation is supported by the National Laboratory of Tsinghua Information Science and Technology.

[1] A. K. Gupta, D. G. Lilley, N. Syred, Swirl flows, Tunbridge Wells, Kent, England, Abacus Press, 1984, 488 p. 1.

[2] Y. Huang, V. Yang, Dynamics and stability of lean-premixed swirlstabilized combustion, Progress in Energy and Combustion Science 35 (4) (2009) 293-364.

[3] Y. Huang, V. Yang, Effect of swirl on combustion dynamics in a leanpremixed swirl-stabilized combustor, Proceedings of the Combustion Institute 30 (2) (2005) 1775-1782.

[4] E. Fernandes, M. Heitor, S. Shtork, An analysis of unsteady highly turbulent swirling flow in a model vortex combustor, Experiments in Fluids 40 (2) (2006) 177-187.

[5] Y. Sommerer, D. Galley, T. Poinsot, S. Ducruix, F. Lacas, D. Veynante, et al., Large eddy simulation and experimental study of flashback and blow-off in a lean partially premixed swirled burner, Journal of Turbulence 5 (37) (2004) 1-3.

[6] P. Weigand, W. Meier, X. Duan, W. Stricker, M. Aigner, Investigations of swirl flames in a gas turbine model combustor: I. flow field, structures, 
temperature, and species distributions, Combustion and flame 144 (1) (2006) 205-224.

[7] W. Meier, X. Duan, P. Weigand, Reaction zone structures and mixing characteristics of partially premixed swirling ch4/air flames in a gas turbine model combustor, Proceedings of the combustion Institute 30 (1) (2005) 835-842.

[8] Y. Huang, V. Yang, Bifurcation of flame structure in a lean-premixed swirl-stabilized combustor: transition from stable to unstable flame, Combustion and Flame 136 (3) (2004) 383-389.

[9] Y. Huang, H.-G. Sung, S.-Y. Hsieh, V. Yang, Large-eddy simulation of combustion dynamics of lean-premixed swirl-stabilized combustor, Journal of Propulsion and Power 19 (5) (2003) 782-794.

[10] S. Prakash, S. Nair, T. Muruganandam, Y. Neumeier, T. Lieuwen, J. M. Seitzman, B. T. Zinn, Acoustic based rapid blowout mitigation in a swirl stabilized combustor, in: ASME Turbo Expo 2005: Power for Land, Sea, and Air, American Society of Mechanical Engineers, 2005, pp. 443-451.

[11] S. Menon, W. Kim, C. Stone, B. Sekar, Large-eddy simulation of fuelair mixing and chemical reactions in swirling flow combustor, AIAA 99 3440.

[12] J. Schluter, Static control of combustion oscillations by coaxial flows: a large-eddy-simulations investigation, Journal of Propulsion and Power 20 (3) (2004) 460-467.

[13] F. Grinstein, C. Fureby, Les studies of the flow in a swirl gas combustor, Proceedings of the combustion institute 30 (2) (2005) 1791-1798.

[14] C. Duwig, L. Fuchs, Study of flame stabilization in a swirling combustor using a new flamelet formulation, Combustion science and technology 177 (8) (2005) 1485-1510.

[15] S. Menon, P.-K. Yeung, W.-W. Kim, Effect of subgrid models on the computed interscale energy transfer in isotropic turbulence, Computers \& fluids 25 (2) (1996) 165-180. 
[16] W. H. Calhoon Jr, On subgrid combustion modeling for large-eddy simulations, Ph.D. thesis, Georgia Institute of Technology (1996).

[17] Y. Zheng, M. Zhu, D. M. Martinez, X. Jiang, Large-eddy simulation of mixing and combustion in a premixed swirling combustor with synthesis gases, Computers \& Fluids 88 (2013) 702-714.

[18] B. F. Magnussen, The Eddy Dissipation Concept for Turbulent Combustion Modelling: Its Physical and Practical Implications, SINTEF, 1990.

[19] C. Fureby, A comparative study of flamelet and finite rate chemistry les for a swirl stabilized flame, Journal of Engineering for Gas Turbines and Power 134 (4) (2012) 041503.

[20] I. V. Novosselov, P. C. Malte, Development and application of an eightstep global mechanism for cfd and crn simulations of lean-premixed combustors, Journal of Engineering for Gas Turbines and Power 130 (2) (2008) 021502.

[21] P. Griebel, P. Siewert, P. Jansohn, Flame characteristics of turbulent lean premixed methane/air flames at high pressure: Turbulent flame speed and flame brush thickness, Proceedings of the Combustion Institute 31 (2) (2007) 3083-3090.

[22] S. Wang, V. Yang, G. Hsiao, S.-Y. Hsieh, H. C. Mongia, Large-eddy simulations of gas-turbine swirl injector flow dynamics, Journal of Fluid Mechanics 583 (2007) 99-122.

[23] F. Biagioli, F. Güthe, Effect of pressure and fuel-air unmixedness on nox emissions from industrial gas turbine burners, Combustion and Flame 151 (1) (2007) 274-288.

\section{Nomenclature}

$\left(\Delta h_{f}^{0}\right)_{m}$ standard heat of formation $(k J / m o l)$

$\Delta \quad$ filtered scale

$\delta_{i j} \quad$ kronecker delta 


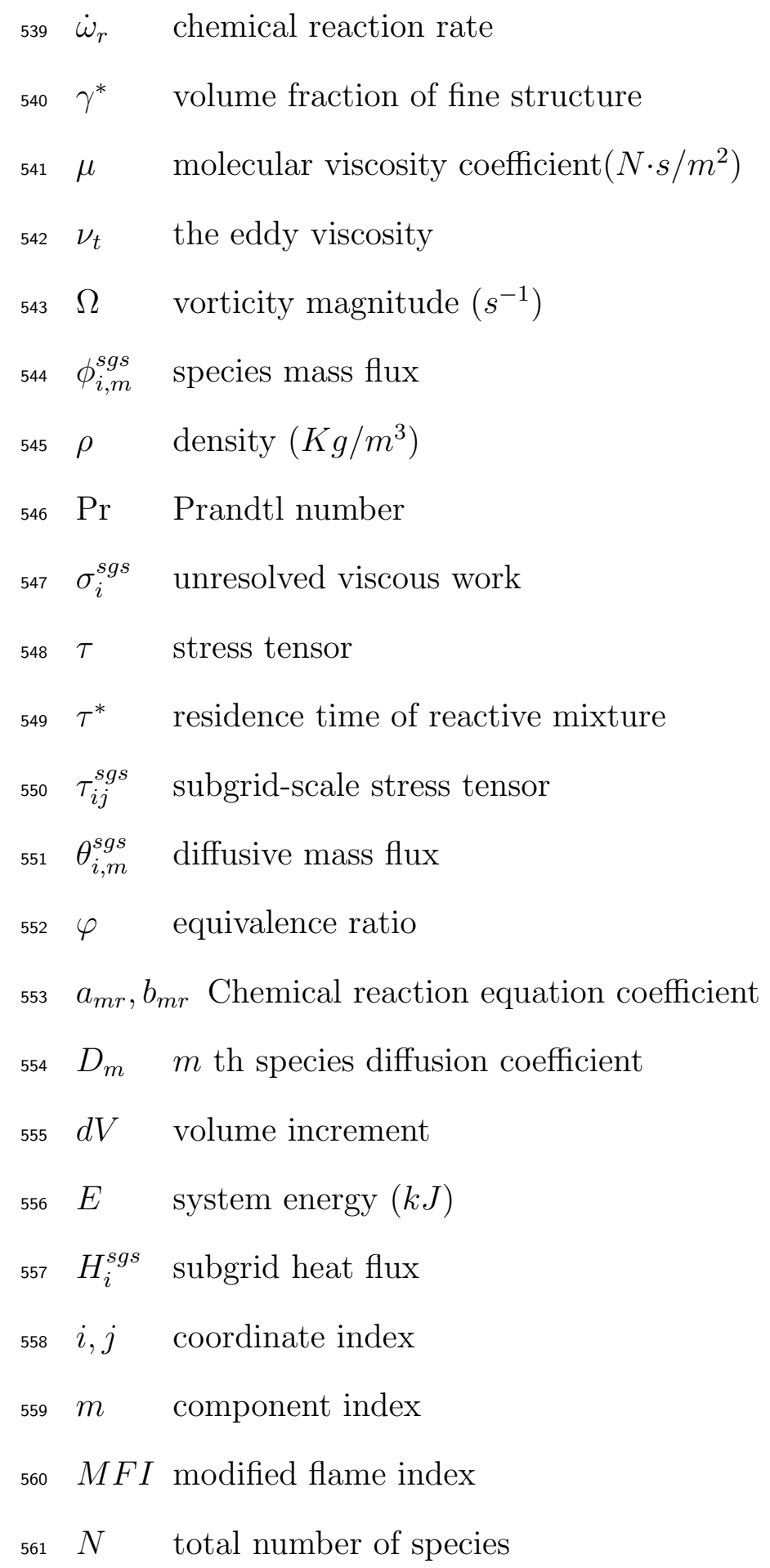




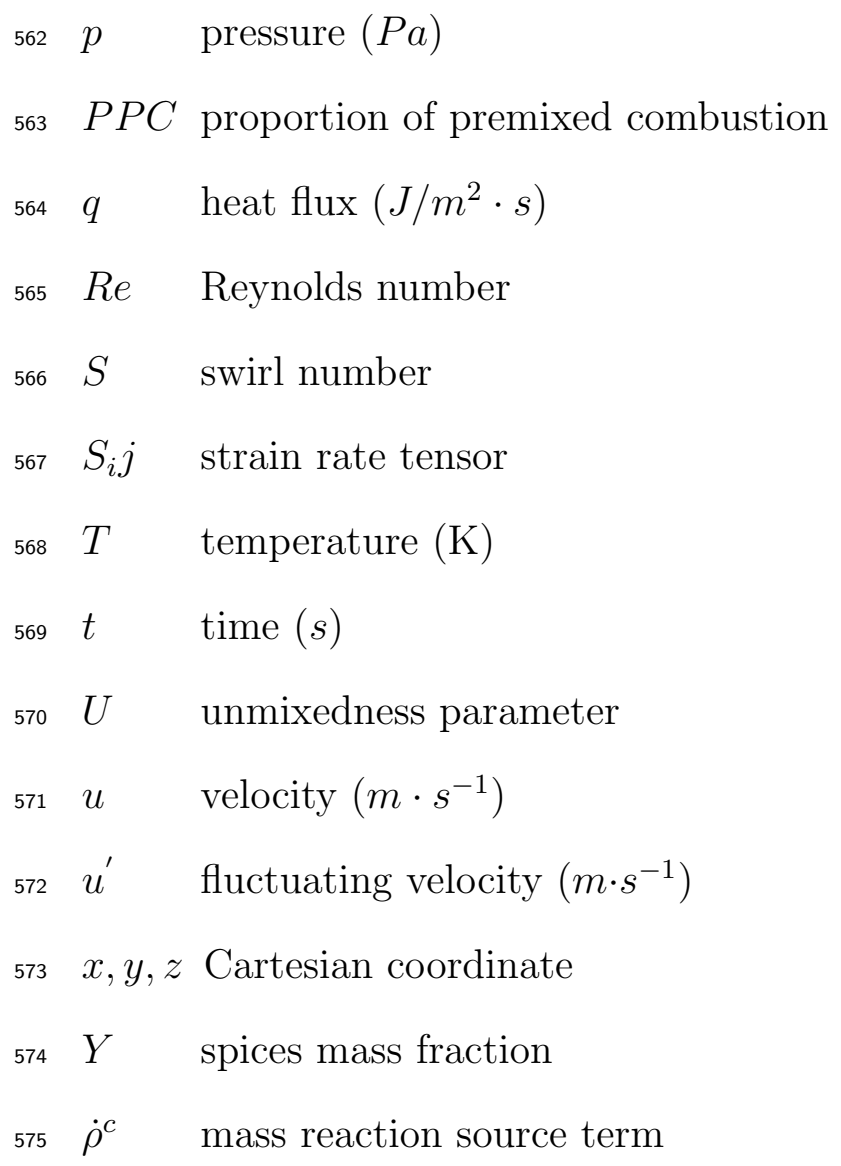

